\title{
Tripled fixed point theorem in fuzzy metric spaces and applications
}

\author{
A Roldán ${ }^{1}$, J Martínez-Moreno ${ }^{2 *}$ and C Roldán ${ }^{3}$
}

${ }^{*}$ Correspondence:

jmmoreno@ujaen.es

2 Department of Mathematics, University of Jaén, Jaén, Spain

Full list of author information is available at the end of the article

\begin{abstract}
In this paper we prove an existence and uniqueness theorem for contractive type mappings in fuzzy metric spaces. In order to do that, we consider a slight modification of the concept of a tripled fixed point introduced by Berinde et al. (Nonlinear Anal. TMA 74:4889-4897, 2011) for nonlinear mappings. Additionally, we obtain some fixed point theorems for metric spaces. These results generalize, extend and unify several classical and very recent related results in literature. For instance, we obtain an extension of Theorem 4.1 in (Zhu and Xiao in Nonlinear Anal. TMA 74:5475-5479, 2011) and a version in non-partially ordered sets of Theorem 2.2 in (Bhaskar and Lakshmikantham in Nonlinear Anal. TMA 65:1379-1393, 2006). As application, we solve a kind of Lipschitzian systems in three variables and an integral system. Finally, examples to support our results are also given.
\end{abstract}

\section{Introduction}

In a recent paper, Bhaskar and Lakshmikantham [1] introduced the concepts of coupled fixed point and mixed monotone property for contractive operators of the form $F: X \times$ $X \rightarrow X$, where $X$ is a partially ordered metric space, and then established some interesting coupled fixed point theorems. They also illustrated these important results by proving the existence and uniqueness of the solution for a periodic boundary value problem. Later, Lakshmikantham and Ćirić [2] proved coupled coincidence and coupled common fixed point results for nonlinear mappings satisfying certain contractive conditions in partially ordered complete metric spaces. After that many results appeared on coupled fixed point theory (see, e.g., [2-8]).

Fixed point theorems have been studied in many contexts, one of which is the fuzzy setting. The concept of fuzzy sets was initially introduced by Zadeh [9] in 1965. To use this concept in topology and analysis, many authors have extensively developed the theory of fuzzy sets and its applications. One of the most interesting research topics in fuzzy topology is to find an appropriate definition of fuzzy metric space for its possible applications in several areas. It is well known that a fuzzy metric space is an important generalization of the metric space. Many authors have considered this problem and have introduced it in different ways. For instance, George and Veeramani [10] modified the concept of a fuzzy metric space introduced by Kramosil and Michalek [11] and defined the Hausdorff topology of a fuzzy metric space. There exists considerable literature about fixed point properties for mappings defined on fuzzy metric spaces, which have been studied by many authors (see [10, 12-16]). Zhu and Xiao [7] and Hu [6] gave a coupled fixed point theorem for contractions in fuzzy metric spaces, and Fang [3] proved some common fixed 
point theorems under $\phi$-contractions for compatible and weakly compatible mappings on Menger probabilistic metric spaces. Moreover, Elagan and Segi Rahmat [17] studied the existence of a fixed point in locally convex topology generated by fuzzy $n$-normed spaces.

Very recently, the concept of tripled fixed point has been introduced by Berinde and Borcut [18]. In their manuscript, some new tripled point theorems are obtained using the mixed $g$-monotone mapping. Their results generalize and extend the Bhaskar and Lakshmikantham's research for nonlinear mappings. Moreover, these results could be used to study the existence of solutions of a periodic boundary value problem involving $y^{\prime \prime}=f\left(t, y, y^{\prime}\right)$. A multidimensional notion of a coincidence point between mappings and some existence and uniqueness fixed points theorems for nonlinear mappings defined on partially ordered metric spaces are studied in [19].

In this paper, our main aim is to obtain an existence and uniqueness theorem for contractive type mappings in the framework of fuzzy metric spaces. In order to do that, we consider a slight modification of the concept of a tripled fixed point introduced by Berinde and Borcut for nonlinear mappings. The power of this result is two-fold. Firstly, we can particularize it to complete metric spaces, obtaining a Berinde-Borcut type result (in nonfuzzy setting). Moreover, our result, in a unified manner, covers also coupled fixed (see Zhu and Xiao [7]) and fixed point theorems. Finally, examples to support our results are also given.

\section{Preliminaries}

Henceforth, $X$ will denote a non-empty set and $X^{3}=X \times X \times X$. Subscripts will be used to indicate the arguments of a function. For instance, $F(x, y, z)$ will be denoted by $F_{x y z}$ and $M(x, y, t)$ will be denoted by $M_{x y}(t)$. Furthermore, for brevity, $g(x)$ will be denoted by $g x$.

A metric on $X$ is a mapping $d: X \times X \rightarrow \mathbb{R}$ satisfying, for all $x, y, z \in X$,
(i)
$d_{x y}=0 \quad$ if and only if $\quad x=y$
(ii) $d_{x y} \leq d_{z x}+d_{z y}$.

From these properties, we can easily deduce that $d_{x y} \geq 0$ and $d_{y x}=d_{x y}$ for all $x, y \in X$. The last requirement is called the triangle inequality. If $d$ is a metric on $X$, we say that $(X, d)$ is a metric space (briefly, a $M S$ ).

Let $(X, d)$ be a MS. A mapping $f: X \rightarrow X$ is said to be Lipschitzian if there exists $k \geq 0$ such that $d\left(f_{x}, f_{y}\right) \leq k d_{x y}$ for all $x, y \in X$. The smallest $k$ (denoted by $k_{f}$ ) for which this inequality holds is said to be the Lipschitz constantfor $f$. A Lipschitzian mapping $f: X \rightarrow X$ is a contraction if $k_{f}<1$.

Theorem 1 (Banach's contraction principle) Every contraction from a complete metric space into itself has a unique fixed point.

If $X=\mathbb{R}$ provided with the Euclidean metric, examples of Lipschitzian mappings $f_{i}: \mathbb{R} \rightarrow$ $\mathbb{R}$ are $f_{1}(x)=K, f_{2}(x)=\alpha x, f_{3}(x)=\sin x, f_{4}(x)=\cos x, f_{5}(x)=\arctan x$ and $f_{6}(x)=1 /\left(1+x^{2}\right)$.

Definition 2 A triangular norm (also called a $t$-norm) is a map $*:[0,1]^{2} \rightarrow[0,1]$ that is associative, commutative, nondecreasing in both arguments and has 1 as identity. For each $a \in[0,1]$, the sequence $\left\{*^{n} a\right\}_{n=1}^{\infty}$ is defined inductively by $*^{1} a=a$ and $*^{n} a=\left(*^{n-1} a\right) * a$. A $t$-norm $*$ is said to be of $H$-type (see [20]) if the sequence $\left\{*^{n} a\right\}_{n=1}^{\infty}$ is equicontinuous at 
$a=1$, i.e., for all $\varepsilon \in(0,1)$, there exists $\eta \in(0,1)$ such that if $a \in(1-\eta, 1]$, then $*^{m} a>1-\varepsilon$ for all $m \in \mathbb{N}$.

The most important and well-known continuous $t$-norm of $H$-type is $*=$ min, that verifies $\min (a, b) \geq a b$ for all $a, b \in[0,1]$. The following result presents a wide range of $t$-norms of $H$-type.

Lemma 3 Let $\delta \in(0,1]$ be a real number and let $*$ be a $t$-norm. Define $*_{\delta}$ as $x *_{\delta} y=x * y$, if $\max (x, y) \leq 1-\delta$, and $x *_{\delta} y=\min (x, y)$, if $\max (x, y)>1-\delta$. Then $*_{\delta}$ is a $t$-norm of H-type.

Definition 4 [11] A triple $(X, M, *)$ is called a fuzzy metric space (in the sense of Kramosil and Michalek; briefly, a FMS) if $X$ is an arbitrary non-empty set, $*$ is a continuous $t$-norm and $M: X \times X \times[0, \infty) \rightarrow[0,1]$ is a fuzzy set satisfying the following conditions, for each $x, y, z \in X$, and $t, s>0$ :

(i) $M_{x y}(0)=0$;

(ii) $M_{x y}(t)=1$ if and only if $x=y$;

(iii) $M_{x y}(t)=M_{y x}(t)$;

(iv) $M_{x y}(\cdot):[0, \infty) \rightarrow[0,1]$ is left continuous;

(v) $M_{x y}(t) * M_{y z}(s) \leq M_{x z}(t+s)$.

In this case, we also say that $(X, M)$ is a $F M S$ under $*$. In the sequel, we will only consider FMS verifying:

(vi) $\lim _{t \rightarrow \infty} M_{x y}(t)=1$ for all $x, y \in X$.

Lemma $5 M_{x y}(\cdot)$ is a non-decreasing function on $[0, \infty)$.

Definition 6 Let $(X, M)$ be a FMS under some $t$-norm. A sequence $\left\{x_{n}\right\} \subset X$ is Cauchy if, for any $\epsilon>0$ and $t>0$, there exists $n_{0} \in \mathbb{N}$ such that $M_{x_{n} x_{m}}(t)>1-\epsilon$ for all $n, m \geq n_{0}$. A sequence $\left\{x_{n}\right\} \subset X$ is convergent to $x \in X$, denoted by $\lim _{n \rightarrow \infty} x_{n}=x$ if, for any $\epsilon>0$ and $t>0$, there exists $n_{0} \in \mathbb{N}$ such that $M_{x_{n} x}(t)>1-\epsilon$ for all $n \geq n_{0}$. A FMS in which every Cauchy sequence is convergent is called complete.

Given any $t$-norm $*$, it is easy to prove that $* \leq \min$. Therefore, if $(X, M)$ is a FMS under min, then $(X, M)$ is a FMS under any (continuous or not) $t$-norm. This is the case in the following examples (in which, obviously, we only define $M_{x y}(t)$ for $t>0$ and $x \neq y$ ).

Example 7 From a metric space $(X, d)$, we can consider a FMS in different ways. For $t>0$ and $x \neq y$, define:

$$
\text { - } M_{x y}^{d}(t)=\frac{t}{t+d_{x y}} . \quad \text { - } M_{x y}^{\mathrm{e}}(t)=\mathrm{e}^{-\frac{d_{x y}}{t}} . \quad \bullet M_{x, y}^{c}(t)= \begin{cases}0, & \text { if } t \leq d_{x y} \\ 1, & \text { if } t>d_{x y} .\end{cases}
$$

It is well known that $\left(X, M^{d}\right)$ is a FMS under the product $*=\cdot$, called the standard FMS on $(X, d)$, since it is the standard way of viewing the metric space $(X, d)$ as a FMS. However, it is also true (though lesser-known) that $\left(X, M^{d}\right),\left(X, M^{\mathrm{e}}\right)$ and $\left(X, M^{c}\right)$ are FMSs under min.

Furthermore, $(X, d)$ is a complete metric space if and only if $\left(X, M^{d}\right)$ (or $\left(X, M^{c}\right)$ or $\left.\left(X, M^{\mathrm{e}}\right)\right)$ is a complete FMS. For instance, this is the case of any non-empty and closed subset (or subinterval) of $\mathbb{R}$ provided with its Euclidean metric. 
Definition 8 A function $g: X \rightarrow X$ on a FMS is said to be continuous at a point $x_{0} \in X$ if, for any sequence $\left\{x_{n}\right\}$ in $X$ converging to $x_{0}$, the sequence $\left\{g x_{n}\right\}$ converges to $g x_{0}$. If $g$ is continuous at each $x \in X$, then $g$ is said to be continuous on $X$. As usual, if $x_{0} \in X$, we will denote $g^{-1}\left(x_{0}\right)=\left\{x \in X: g x=x_{0}\right\}$.

Remark 9 If $x \in[0,1]$ and $a, b \in(0, \infty)$, then $a \leq b$ implies that $x^{a} \geq x^{b}$. We will use this fact in the following way: $0<a \leq b \leq 1$ implies that $M_{x y}(t)^{a} \geq M_{x y}(t)^{b} \geq M_{x y}(t)$.

\section{The main result}

Definition 10 Let $F: X^{3} \rightarrow X$ and $g: X \rightarrow X$ be two mappings.

- We say that $F$ and $g$ are commuting if $g F_{x y z}=F_{\text {gxgygz }}$ for all $x, y, z \in X$.

- A point $(x, y, z) \in X^{3}$ is called a tripled coincidence point of the mappings $F$ and $g$ if $F_{x y z}=g x, F_{y z x}=g y$ and $F_{z x y}=g z$.

Theorem 11 Let $*$ be a $t$-norm of $H$-type such that $s * t \geq$ st for all $s, t \in[0,1]$. Let $k \in$ $(0,1)$ and $a, b, c \in[0,1]$ be real numbers such that $a+b+c \leq 1$, let $(X, M, *)$ be a complete $F M S$ and let $F: X^{3} \rightarrow X$ and $g: X \rightarrow X$ be two mappings such that $F\left(X^{3}\right) \subseteq g(X)$ and $g$ is continuous and commuting with $F$. Suppose that for all $x, y, z, u, v, w \in X$ and all $t>0$,

$$
M_{F_{x y z} F_{u v w}}(k t) \geq M_{g x g u}(t)^{a} * M_{g y g v}(t)^{b} * M_{g z g w}(t)^{c} .
$$

Then there exists a unique $x \in X$ such that $x=g x=F_{x x x}$. In particular, $F$ and $g$ have, at least, one tripled coincidence point. Furthermore, $(x, x, x)$ is the unique tripled coincidence point of $F$ and $g$ if we assume that $g^{-1}\left(x_{0}\right)=\left\{x_{0}\right\}$ only in the case that $F \equiv x_{0}$ is constant on $X^{3}$.

In this result, in order to avoid the indetermination $0^{0}$, we assume that $M_{g x g u}(t)^{0}=1$ for all $t>0$ and all $x, y \in X$.

Proof Suppose that $F$ is constant in $X^{3}$, i.e., there exists $x_{0} \in X$ such that $F_{x y z}=x_{0}$ for all $x, y, z \in X$. As $F$ and $g$ are commuting, we deduce that $g x_{0}=g F_{x y z}=F_{g x g y g z}=x_{0}$. Therefore, $x_{0}=g x_{0}=F_{x_{0} x_{0} x_{0}}$ and $\left(x_{0}, x_{0}, x_{0}\right)$ is a tripled coincidence point of $F$ and $g$. Now, suppose that $g^{-1}\left(x_{0}\right)=\left\{x_{0}\right\}$ and $(x, y, z) \in X^{3}$ is another tripled coincidence point of $F$ and $g$. Then $g x=F_{x y z}=x_{0}$, so $x \in g^{-1}\left(x_{0}\right)=\left\{x_{0}\right\}$. Similarly, $x=y=z=x_{0}$ and $\left(x_{0}, x_{0}, x_{0}\right)$ is the unique tripled coincidence point of $F$ and $g$.

Next, suppose that $F$ is not constant in $X^{3}$. In this case, $(a, b, c) \neq(0,0,0)$ and the proof is divided into five steps. Throughout this proof, $n$ and $p$ will denote non-negative integers and $t \in[0, \infty)$.

Step 1. Definition of the sequences $\left\{x_{n}\right\},\left\{y_{n}\right\}$ and $\left\{z_{n}\right\}$. Let $x_{0}, y_{0}, z_{0} \in X$ be three arbitrary points of $X$. Since $F\left(X^{3}\right) \subseteq g(X)$, we can choose $x_{1}, y_{1}, z_{1} \in X$ such that $g x_{1}=F_{x_{0} y_{0} z_{0}}$, $g y_{1}=F_{y_{0} z_{0} x_{0}}$ and $g z_{1}=F_{z_{0} x_{0} y_{0}}$. Again, from $F\left(X^{3}\right) \subseteq g(X)$, we can choose $x_{2}, y_{2}, z_{2} \in X$ such that $g x_{2}=F_{x_{1} y_{1} z_{1}}, g y_{2}=F_{y_{1} z_{1} x_{1}}$ and $g z_{2}=F_{z_{1} x_{1} y_{1}}$. Continuing this process, we can construct sequences $\left\{x_{n}\right\},\left\{y_{n}\right\}$ and $\left\{z_{n}\right\}$ such that, for $n \geq 0, g x_{n+1}=F_{x_{n} y_{n} z_{n}}, g y_{n+1}=F_{y_{n} z_{n} x_{n}}$ and $g z_{n+1}=F_{z_{n} x_{n} y_{n}}$.

Step 2. $\left\{g x_{n}\right\},\left\{g y_{n}\right\}$ and $\left\{g z_{n}\right\}$ are Cauchy sequences. Define, for $n \geq 0$ and all $t \geq 0, \delta_{n}(t)=$ $M_{g x_{n} g x_{n+1}}(t) * M_{g y_{n} g y_{n+1}}(t) * M_{g z_{n} g_{z n+1}}(t)$. Since $\delta_{n}$ is a non-decreasing function and $t-k t \leq$ 
$t \leq t / k$, we have that

$$
\delta_{n}(t-k t) \leq \delta_{n}(t) \leq \delta_{n}(t / k) \text { for all } t>0 \text { and } n \geq 0 \text {. }
$$

From inequality (1) we deduce, for all $n \in \mathbb{N}$ and all $t \geq 0$,

$$
\begin{aligned}
M_{g x_{n} g x_{n+1}}(t) & =M_{F_{x_{n-1} y_{n-1} z_{n-1}} F_{x_{n} y_{n} z_{n}}(t)} \\
& \geq M_{g x_{n-1} g x_{n}}\left(\frac{t}{k}\right)^{a} * M_{g y_{n-1} g y_{n}}\left(\frac{t}{k}\right)^{b} * M_{g z_{n-1} g z_{n}}\left(\frac{t}{k}\right)^{c} ; \\
M_{g y_{n} g y_{n+1}}(t) & =M_{F_{y_{n-1} z_{n-1} x_{n-1}} F_{y_{n} z_{n} x_{n}}(t)} \\
& \geq M_{g y_{n-1} g y_{n}}\left(\frac{t}{k}\right)^{a} * M_{g z_{n-1} g z_{n}}\left(\frac{t}{k}\right)^{b} * M_{g x_{n-1} g x_{n}}\left(\frac{t}{k}\right)^{c} ; \\
M_{g z_{n} g z_{n+1}}(t) & =M_{F_{z_{n-1} x_{n-1} y_{n-1}} F_{z_{n} x_{n} y_{n}}(t)} \\
& \geq M_{g z_{n-1} g z_{n}}\left(\frac{t}{k}\right)^{a} * M_{g x_{n-1} g x_{n}}\left(\frac{t}{k}\right)^{b} * M_{g y_{n-1} g y_{n}}\left(\frac{t}{k}\right)^{c} .
\end{aligned}
$$

According to (3), (4), (5) and Remark 9, we have that

$$
\begin{aligned}
M_{g x_{n} g x_{n+1}}(t) & \geq M_{g x_{n-1} g x_{n}}(t / k)^{a} * M_{g y_{n-1} g y_{n}}(t / k)^{b} * M_{g z_{n-1} g z_{n}}(t / k)^{c} \\
& \geq M_{g x_{n-1} g x_{n}}(t / k) * M_{g y_{n-1} g y_{n}}(t / k) * M_{g z_{n-1} g z_{n}}(t / k)=\delta_{n-1}(t / k) ; \\
M_{g y_{n} g y_{n+1}}(t) & \geq M_{g y_{n-1} g y_{n}}(t / k)^{a} * M_{g z_{n-1} g z_{n}}(t / k)^{b} * M_{g x_{n-1} g x_{n}}(t / k)^{c} \\
& \geq M_{g y_{n-1} g y_{n}}(t / k) * M_{g z_{n-1} g z_{n}}(t / k) * M_{g x_{n-1} g x_{n}}(t / k)=\delta_{n-1}(t / k) ; \\
M_{g z_{n} g z_{n+1}}(t) & \geq M_{g z_{n-1} g z_{n}}(t / k)^{a} * M_{g x_{n-1} g x_{n}}(t / k)^{b} * M_{g y_{n-1} g y_{n}}(t / k)^{c} \\
& \geq M_{g z_{n-1} g z_{n}}(t / k) * M_{g x_{n-1} g x_{n}}(t / k) * M_{g y_{n-1} g y_{n}}(t / k)=\delta_{n-1}(t / k) .
\end{aligned}
$$

This proves that, for all $t>0$ and all $n \geq 0$,

$$
M_{g x_{n} g x_{n+1}}(t), M_{g y_{n} g y_{n+1}}(t), M_{g z_{n} g z_{n+1}}(t) \geq \delta_{n-1}(t / k) \geq \delta_{n-1}(t) .
$$

Swapping $t$ by $t-k t$, we deduce, for all $t>0$ and $n \geq 0$, that

$$
M_{g x_{n} g x_{n+1}}(t-k t), M_{g y_{n} g y_{n+1}}(t-k t), M_{g z_{n} g z_{n+1}}(t-k t) \geq \delta_{n-1}(t-k t) .
$$

Taking into account that $*$ is commutative and $* \geq \cdot$, and (3), (4), (5), we observe that

$$
\begin{aligned}
\delta_{n}(t)= & M_{g x_{n} g x_{n+1}}(t) * M_{g y_{n} g y_{n+1}}(t) * M_{g z_{n} g z_{n+1}}(t) \\
\geq & \left(M_{g x_{n-1} g x_{n}}(t / k)^{a} * M_{g y_{n-1} g y_{n}}(t / k)^{b} * M_{g z_{n-1} g z_{n}}(t / k)^{c}\right) \\
& *\left(M_{g x_{n-1} g x_{n}}(t / k)^{c} * M_{g y_{n-1} g y_{n}}(t / k)^{a} * M_{g z_{n-1} g z_{n}}(t / k)^{b}\right) \\
& *\left(M_{g x_{n-1} g x_{n}}(t / k)^{b} * M_{g y_{n-1} g y_{n}}(t / k)^{c} * M_{g z_{n-1} g z_{n}}(t / k)^{a}\right) \\
= & \left(M_{g x_{n-1} g x_{n}}(t / k)^{a} * M_{g x_{n-1} g x_{n}}(t / k)^{c} * M_{g x_{n-1} g x_{n}}(t / k)^{b}\right) \\
& *\left(M_{g y_{n-1} g y_{n}}(t / k)^{b} * M_{g y_{n-1} g y_{n}}(t / k)^{a} * M_{g x_{n-1} g x_{n}}(t / k)^{c}\right)
\end{aligned}
$$




$$
\begin{aligned}
& *\left(M_{g z_{n-1} g z_{n}}(t / k)^{c} * M_{g z_{n-1} g z_{n}}(t / k)^{b} * M_{g z_{n-1} g z_{n}}(t / k)^{a}\right) \\
\geq & \left(M_{g x_{n-1} g x_{n}}(t / k)^{a} \cdot M_{g x_{n-1} g x_{n}}(t / k)^{c} \cdot M_{g x_{n-1} g x_{n}}(t / k)^{b}\right) \\
& *\left(M_{g y_{n-1} g y_{n}}(t / k)^{b} \cdot M_{g y_{n-1} g y_{n}}(t / k)^{a} \cdot M_{g x_{n-1} g x_{n}}(t / k)^{c}\right) \\
& *\left(M_{g z_{n-1} g z_{n}}(t / k)^{c} \cdot M_{g z_{n-1} g z_{n}}(t / k)^{b} \cdot M_{g z_{n-1} g z_{n}}(t / k)^{a}\right) \\
= & M_{g x_{n-1} g x_{n}}(t / k)^{a+b+c} * M_{g y_{n-1} g y_{n}}(t / k)^{a+b+c} * M_{g z_{n-1} g z_{n}}(t / k)^{a+b+c} \\
\geq & M_{g x_{n-1} g x_{n}}(t / k) * M_{g y_{n-1} g y_{n}}(t / k) * M_{g z_{n-1} g z_{n}}(t / k)=\delta_{n-1}(t / k) .
\end{aligned}
$$

If we join this property to (2),

$$
\delta_{n}(t) \geq \delta_{n-1}(t / k) \geq \delta_{n-1}(t) \geq \delta_{n-1}(t-k t) \quad \text { for all } t>0 \text { and } n \geq 1
$$

Repeatedly applying the first inequality, we deduce that $\delta_{n}(t) \geq \delta_{n-1}(t / k) \geq \delta_{n-2}\left(t / k^{2}\right) \geq$ $\cdots \geq \delta_{0}\left(t / k^{n}\right)$ for all $t>0$ and $n \geq 1$. This means that for all $t>0$,

$$
\lim _{n \rightarrow \infty} \delta_{n}(t) \geq \lim _{n \rightarrow \infty} \delta_{0}\left(t / k^{n}\right)=1 \Rightarrow \lim _{n \rightarrow \infty} \delta_{n}(t)=1
$$

Properties (6) and (8) imply that

$$
M_{g x_{n} g x_{n+1}}(t), M_{g y_{n} g y_{n+1}}(t), M_{g z_{n} g z_{n+1}}(t) \geq \delta_{n}(t) \geq \delta_{n-1}(t-k t) .
$$

Next, we claim that

$$
M_{g x_{n} g x_{n+p}}(t), M_{g y_{n} g y_{n+p}}(t), M_{g z_{n} g z_{n+p}}(t) \geq *^{p} \delta_{n-1}(t-k t) \quad \text { for all } t>0, n, p \geq 1
$$

We prove it by induction methodology in $p \geq 1$. If $p=1$, (11) is true for all $n \geq 1$ and all $t>0$ by (10). Suppose that (11) is true for all $n \geq 1$ and all $t>0$ for some $p$, and we are going to prove it for $p+1$. Applying (1), the induction hypothesis and that $* \geq$,

$$
\begin{aligned}
M_{g x_{n+1} g x_{n+p+1}}(k t) & =M_{F_{x_{n} y_{n} z_{n}} F_{x_{n+p} y_{n+p} z_{n+p}}}(k t) \\
& \geq M_{g x_{n} g x_{n+p}}(t)^{a} * M_{g y_{n} g y_{n+p}}(t)^{b} * M_{g z_{n} g z_{n+p}}(t)^{c} \\
& \geq\left(*^{p} \delta_{n-1}(t-k t)\right)^{a} *\left(*^{p} \delta_{n-1}(t-k t)\right)^{b} *\left(*^{p} \delta_{n-1}(t-k t)\right)^{c} \\
& \geq\left(*^{p} \delta_{n-1}(t-k t)\right)^{a} \cdot\left(*^{p} \delta_{n-1}(t-k t)\right)^{b} \cdot\left(*^{p} \delta_{n-1}(t-k t)\right)^{c} \\
& =\left(*^{p} \delta_{n-1}(t-k t)\right)^{a+b+c} \geq *^{p} \delta_{n-1}(t-k t) .
\end{aligned}
$$

Arguing in the same way, we come to $M_{g x_{n+1} g x_{n+1+p}}(k t), M_{g y_{n+1} g y_{n+1+p}}(k t), M_{g z_{n+1} g z_{n+1+p}}(k t) \geq$ $*^{p} \delta_{n-1}(t-k t)$. Applying the axiom (v) of a FMS, (7) and the induction hypothesis,

$$
\begin{aligned}
M_{g x_{n} g x_{n+p+1}}(t) & =M_{g x_{n} g x_{n+p+1}}(t-k t+k t) \\
& \geq M_{g x_{n} g x_{n+1}}(t-k t) * M_{g x_{n+1} g x_{n+1+p}}(k t) \\
& \geq \delta_{n-1}(t-k t) *\left(*^{p} \delta_{n-1}(t-k t)\right)=*^{p+1} \delta_{n-1}(t-k t) .
\end{aligned}
$$


The same reasoning is also valid for $M_{g y_{n} g y_{n+p+1}}(t)$ and $M_{g z_{n} g z_{n+p+1}}(t)$. Therefore, (11) is true. This permits us to show that $\left\{g x_{n}\right\}$ is Cauchy. Suppose that $t>0$ and $\varepsilon \in(0,1)$ are given. By the hypothesis, as $*$ is a $t$-norm of $H$-type, there exists $0<\eta<1$ such that $*^{p} a>1-\varepsilon$ for all $a \in(1-\eta, 1]$ and for all $p \geq 1$. By $(9), \lim _{n \rightarrow \infty} \delta_{n}(t)=1$, so there exists $n_{0} \in \mathbb{N}$ such that $\delta_{n}(t-k t)>1-\eta$ for all $n \geq n_{0}$. Hence (11), we get $M_{g x_{n} g x_{n+p}}(t), M_{g y_{n} g y_{n+p}}(t), M_{g z_{n} g z_{n+p}}(t)>1-\varepsilon$ for all $n \geq n_{0}$ and $p \geq 1$. Therefore, $\left\{g x_{n}\right\}$ is a Cauchy sequence. Similarly, $\left\{g y_{n}\right\}$ and $\left\{g z_{n}\right\}$ are also Cauchy sequences.

Step 3. We claim that $g$ and $F$ have a tripled coincidence point. Since $X$ is complete, there exist $x, y, z \in X$ such that $\lim _{n \rightarrow \infty} g x_{n}=x, \lim _{n \rightarrow \infty} g y_{n}=y$ and $\lim _{n \rightarrow \infty} g z_{n}=z$. As $g$ is continuous, we have that $\lim _{n \rightarrow \infty} g g x_{n}=g x, \lim _{n \rightarrow \infty} g g y_{n}=g y$ and $\lim _{n \rightarrow \infty} g g z_{n}=g z$. The commutativity of $F$ with $g$ implies that $g g x_{n+1}=g F\left(x_{n}, y_{n}, z_{n}\right)=F\left(g x_{n}, g y_{n}, g z_{n}\right)$. By (1),

$$
\begin{aligned}
M_{g g x_{n+1} F_{x y z}}(k t) & =M_{F_{g x n g y n g z n} F_{x y z}}(k t) \geq M_{g g x_{n} g x}(t)^{a} * M_{g g y_{n} g y}(t)^{b} * M_{g g z_{n} g z}(t)^{c} \\
& \geq M_{g g x_{n} g x}(t) * M_{g g y_{n} g y}(t) * M_{g g z_{n} g z}(t) .
\end{aligned}
$$

Letting $n \rightarrow \infty$, we deduce that $\lim _{n \rightarrow \infty} g g x_{n}=F_{x y z}$. Hence, $F_{x y z}=g x$. In a similar way, we can show that $F_{y z x}=g y$ and $F_{z x y}=g z$, so $(x, y, z)$ is a tripled coincidence point of the mappings $F$ and $g$.

$$
F_{x y z}=g x, \quad F_{y z x}=g y \quad \text { and } \quad F_{z x y}=g z .
$$

Step 4. We claim that $x=F_{z x y}, y=F_{x y z}$ and $z=F_{y z x}$. We note that by condition (1),

$$
\begin{aligned}
& M_{g x g y_{n+1}}(k t)=M_{F_{x y z} F_{y_{n} z_{n} x_{n}}}(k t) \geq M_{g x g y_{n}}(t)^{a} * M_{g y g z_{n}}(t)^{b} * M_{g z g x_{n}}(t)^{c} ; \\
& M_{g y g z_{n+1}}(k t)=M_{F_{y z x} F_{z_{n} x_{n} y_{n}}}(k t) \geq M_{g y g z_{n}}(t)^{a} * M_{g z g x_{n}}(t)^{b} * M_{g x g y_{n}}(t)^{c} ; \\
& M_{g z g x_{n+1}}(k t)=M_{F_{z x y} F_{x_{n} y_{n} z_{n}}}(k t) \geq M_{g z g x_{n}}(t)^{a} * M_{g x g y_{n}}(t)^{b} * M_{g y g z_{n}}(t)^{c} .
\end{aligned}
$$

Let $\beta_{n}(t)=M_{g x g y_{n}}(t) * M_{g y g z_{n}}(t) * M_{g z g x_{n}}(t)$ for all $t>0$ and $n \geq 0$. By (13), (14) and (15),

$$
\begin{aligned}
\beta_{n+1}(k t)= & M_{g x g y_{n+1}}(k t) * M_{g y g z_{n+1}}(k t) * M_{g z g x_{n}}(k t) \\
\geq & \left(M_{g x g y_{n}}(t)^{a} * M_{g y g z_{n}}(t)^{b} * M_{g z g x_{n}}(t)^{c}\right) \\
& *\left(M_{g y g z_{n}}(t)^{a} * M_{g z g x_{n}}(t)^{b} * M_{g x g y_{n}}(t)^{c}\right) \\
& *\left(M_{g z g x_{n}}(t)^{a} * M_{g x g y_{n}}(t)^{b} * M_{g y g z_{n}}(t)^{c}\right) \\
= & \left(M_{g x g y_{n}}(t)^{a} * M_{g x g y_{n}}(t)^{c} * M_{g x g y_{n}}(t)^{b}\right) \\
& *\left(M_{g y g z_{n}}(t)^{b} * M_{g y g z_{n}}(t)^{a} * M_{g y g z_{n}}(t)^{c}\right) \\
& *\left(M_{g z g x_{n}}(t)^{c} * M_{g z g x_{n}}(t)^{b} * M_{g z g x_{n}}(t)^{a}\right) \\
\geq & \left(M_{g x g y_{n}}(t)^{a} \cdot M_{g x g y_{n}}(t)^{c} \cdot M_{g x g y_{n}}(t)^{b}\right) \\
& *\left(M_{g y g z_{n}}(t)^{b} \cdot M_{g y g z_{n}}(t)^{a} \cdot M_{g y g z_{n}}(t)^{c}\right) \\
& *\left(M_{g z g x_{n}}(t)^{c} \cdot M_{g z g x_{n}}(t)^{b} \cdot M_{g z g x_{n}}(t)^{a}\right) \\
= & M_{g x g y_{n}}(t)^{a+b+c} * M_{g y g z_{n}}(t)^{a+b+c} * M_{g z g x_{n}}(t)^{a+b+c} \\
\geq & M_{g x g y_{n}}(t) * M_{g y g z_{n}}(t) * M_{g z g x_{n}}(t)=\beta_{n}(t) .
\end{aligned}
$$


This proves that $\beta_{n+1}(k t) \geq \beta_{n}(t)$ for all $n \geq 0$ and all $t>0$. Repeating this process,

$$
\beta_{n}(t) \geq \beta_{n-1}(t / k) \geq \beta_{n-2}\left(t / k^{2}\right) \geq \cdots \geq \beta_{0}\left(t / k^{n}\right) \quad \text { for all } t>0 \text { and } n \geq 1
$$

Now, by (16), (13), (14) and (15),

$$
\begin{aligned}
& M_{g x g y_{n+1}}(k t) \geq M_{g x g y_{n}}(t)^{a} * M_{g y g z_{n}}(t)^{b} * M_{g z g x_{n}}(t)^{c} \geq \beta_{n}(t) \geq \beta_{0}\left(t / k^{n}\right) ; \\
& M_{g y g z_{n+1}}(k t) \geq M_{g y g z_{n}}(t)^{a} * M_{g z g x_{n}}(t)^{b} * M_{g x g y_{n}}(t)^{c} \geq \beta_{n}(t) \geq \beta_{0}\left(t / k^{n}\right) ; \\
& M_{g z g x_{n+1}}(k t) \geq M_{g z g x_{n}}(t)^{a} * M_{g x g y_{n}}(t)^{b} * M_{g y g z_{n}}(t)^{c} \geq \beta_{n}(t) \geq \beta_{0}\left(t / k^{n}\right) .
\end{aligned}
$$

Therefore, $M_{g x g y_{n+1}}(k t), M_{g y g z_{n+1}}(k t), M_{g z g x_{n+1}}(k t) \geq \beta_{0}\left(t / k^{n}\right)$ for all $t>0$ and $n \geq 1$. Since $\lim _{n \rightarrow \infty} \beta_{0}\left(t / k^{n}\right)=1$ for all $t>0$, we have, taking limit in (17), (18) and (19), that $\lim _{n \rightarrow \infty} g x_{n}=g z, \lim _{n \rightarrow \infty} g y_{n}=g x$ and $\lim _{n \rightarrow \infty} g z_{n}=g y$. This shows, using (12), that

$$
F_{x y z}=g x=\lim _{n \rightarrow \infty} g y_{n}=y, \quad F_{y z x}=g y=\lim _{n \rightarrow \infty} g z_{n}=z, \quad F_{z x y}=g z=\lim _{n \rightarrow \infty} g x_{n}=x .
$$

Step 5. We will prove that $x=y=z$. Let $\theta(t)=M_{x y}(t) * M_{y z}(t) * M_{z x}(t)$ for all $t>0$. Then, by condition (1),

$$
\begin{aligned}
M_{x y}(k t) & =M_{F_{x y z} F_{y z x}}(k t) \geq M_{g x g y}(t)^{a} * M_{g y g z}(t)^{b} * M_{g z g x}(t)^{c} \\
& =M_{y z}(t)^{a} * M_{z x}(t)^{b} * M_{x y}(t)^{c} ; \\
M_{y z}(k t) & =M_{F_{y z x} F_{z x y}}(k t) \geq M_{g y g z}(t)^{a} * M_{g z g x}(t)^{b} * M_{g x g y}(t)^{c} \\
& =M_{z x}(t)^{a} * M_{x y}(t)^{b} * M_{y z}(t)^{c} ; \\
M_{z x}(k t) & =M_{F_{z x y} F_{x y z}}(k t) \geq M_{g z g x}(t)^{a} * M_{g x g y}(t)^{b} * M_{g y g z}(t)^{c} \\
& =M_{x y}(t)^{a} * M_{y z}(t)^{b} * M_{z x}(t)^{c} .
\end{aligned}
$$

If we use these three inequalities at the same time,

$$
\begin{aligned}
\theta(k t)= & M_{x y}(k t) * M_{y z}(k t) * M_{z x}(k t) \\
\geq & \left(M_{y z}(t)^{a} * M_{z x}(t)^{b} * M_{x y}(t)^{c}\right) *\left(M_{z x}(t)^{a} * M_{x y}(t)^{b} * M_{y z}(t)^{c}\right) \\
& *\left(M_{x y}(t)^{a} * M_{y z}(t)^{b} * M_{z x}(t)^{c}\right) \\
= & \left(M_{x y}(t)^{c} * M_{x y}(t)^{b} * M_{x y}(t)^{a}\right) *\left(M_{y z}(t)^{a} * M_{y z}(t)^{c} * M_{y z}(t)^{b}\right) \\
& *\left(M_{z x}(t)^{b} * M_{z x}(t)^{a} * M_{z x}(t)^{c}\right) \\
\geq & \left(M_{x y}(t)^{c} \cdot M_{x y}(t)^{b} \cdot M_{x y}(t)^{a}\right) *\left(M_{y z}(t)^{a} \cdot M_{y z}(t)^{c} \cdot M_{y z}(t)^{b}\right) \\
& *\left(M_{z x}(t)^{b} \cdot M_{z x}(t)^{a} \cdot M_{z x}(t)^{c}\right) \\
= & M_{x y}(t)^{a+b+c} * M_{y z}(t)^{a+b+c} * M_{z x}(t)^{a+b+c} \\
\geq & M_{x y}(t) * M_{y z}(t) * M_{z x}(t)=\theta(t) .
\end{aligned}
$$


We find that $\theta(k t) \geq \theta(t)$ implies that $\theta(t) \geq \theta(t / k) \geq \theta\left(t / k^{2}\right) \geq \cdots \geq \theta\left(t / k^{n}\right)$ for all $t>0$ and $n \geq 1$. By (20), (21) and (22),

$$
\begin{aligned}
& M_{x y}(k t) \geq M_{y z}(t)^{a} * M_{z x}(t)^{b} * M_{x y}(t)^{c} \geq M_{y z}(t) * M_{z x}(t) * M_{x y}(t)=\theta(t) \geq \theta\left(t / k^{n}\right), \\
& M_{y z}(k t) \geq M_{z x}(t)^{a} * M_{x y}(t)^{b} * M_{y z}(t)^{c} \geq M_{z x}(t) * M_{x y}(t) * M_{y z}(t)=\theta(t) \geq \theta\left(t / k^{n}\right), \\
& M_{z x}(k t) \geq M_{x y}(t)^{a} * M_{y z}(t)^{b} * M_{z x}(t)^{c} \geq M_{x y}(t) * M_{y z}(t) * M_{z x}(t)=\theta(t) \geq \theta\left(t / k^{n}\right) .
\end{aligned}
$$

Letting $n \rightarrow \infty$, we have $\lim _{n \rightarrow \infty} \theta\left(t / k^{n}\right)=1$ for all $t>0$, and this means that $M_{x y}(k t)=$ $M_{y z}(k t)=M_{z x}(k t)=1$ for all $t>0$, i.e., $x=y=z$. The unicity of $x$ follows from (1).

Remark 12 The unicity of the coincidence point of $F$ and $g$ is not always true. For instance, if $F \equiv x_{0}$ is constant and $g \equiv x_{0}$ is also constant, then every $(x, y, z) \in X^{3}$ is a coincidence point of $F$ and $g$.

Remark 13 In the previous theorem, we have only used the continuity of $*$ at $(1,1)$, that is, if $\left\{x_{n}\right\},\left\{y_{n}\right\} \subset[0,1]$ are sequences such that $\left\{x_{n}\right\} \rightarrow 1$ and $\left\{y_{n}\right\} \rightarrow 1$, then $\left\{x_{n} * y_{n}\right\} \rightarrow 1$. And this is true because $\left\{x_{n} * y_{n}\right\} \geq\left\{x_{n} \cdot y_{n}\right\} \rightarrow 1 \cdot 1=1$.

Example 14 Consider $\left(X=\mathbb{R}, M^{\mathrm{e}}\right)$ as in Example 7. Let $\alpha, \beta>0$ and $k \in(0,1)$ be positive real numbers such that $6 \alpha \leq \beta k$ (in particular, $\alpha / k \leq \beta / 6$ ). Define $F: \mathbb{R}^{3} \rightarrow \mathbb{R}$ and $g: \mathbb{R} \rightarrow$ $\mathbb{R}$ as $F(x, y, z)=\alpha(x-y)$ and $g x=\beta x$ for all $x, y, z \in X$. Clearly, $g$ is continuous, $F$ and $g$ are commuting and $F\left(\mathbb{R}^{3}\right)=\mathbb{R}=g(\mathbb{R})$. We also note that $M^{\mathrm{e}}$ verifies

$$
\begin{aligned}
M_{F_{x y z} F_{u v w}}^{\mathrm{e}}(k t) & =\left(\mathrm{e}^{|(x-u)+(v-y)|}\right)^{-\frac{\alpha}{k t}} \geq\left(\mathrm{e}^{-\frac{2 \max (|x-u||| v-y \mid)}{t}}\right)^{\frac{\alpha}{k}} \\
& \geq\left(\mathrm{e}^{-\frac{2 \max (|x-u||| v-y \mid)}{t}}\right)^{\frac{\beta}{6}}=\left(\mathrm{e}^{-\frac{\beta}{3 t}}\right)^{\max (|x-u|,|v-y|)}=\min \left(\mathrm{e}^{-\frac{\beta|x-u|}{3 t}}, \mathrm{e}^{-\frac{\beta|v-y|}{3 t}}\right) \\
& \geq \min \left(\mathrm{e}^{-\frac{|\beta x-\beta u|}{3 t}}, \mathrm{e}^{-\frac{|\beta y-\beta v|}{3 t}}, \mathrm{e}^{-\frac{|\beta z-\beta w|}{3 t}}\right) \\
& =\min \left(\left[M_{g x g u}^{\mathrm{e}}(t)\right]^{1 / 3},\left[M_{g y g \nu}^{\mathrm{e}}(t)\right]^{1 / 3},\left[M_{g z g w}^{\mathrm{e}}(t)\right]^{1 / 3}\right) .
\end{aligned}
$$

Therefore, applying Theorem 11, we deduce that $F$ and $g$ have a tripled coincidence point.

\section{Consequences}

In the proof of the next result, the view of $(X, d)$ as the crisp FMS $\left(X, M^{c}, \min \right)$ is used (see Example 7). This approach allows us to deduce results for metric spaces from the corresponding result in the fuzzy setting. Moreover, Theorem 15 is just a tripled coincidence point result, similar to Berinde-Borcut one, see [18, Theorem 7] and [21, Theorem 4], in a not necessarily partially ordered set.

Theorem 15 Let $(X, d)$ be a complete metric space and let $F: X^{3} \rightarrow X$ and $g: X \rightarrow X$ be two mappings such that $F\left(X^{3}\right) \subseteq g(X)$ and $g$ is continuous and commuting with $F$. Suppose that $F$ and $g$ verify some of the following conditions for all $x, y, z, u, v, w \in X$ :

(a) $d_{F_{x y z} F_{u v w}} \leq k \max \left(d_{g x g u}, d_{g y g v}, d_{g z g w}\right)$ for some $k \in(0,1)$.

(b) $d_{F_{x y z} F_{u v w}} \leq k\left(\alpha d_{g x g u}+\beta d_{g y g v}+\gamma d_{g z g w}\right)$ for some $k \in(0,1)$ and some $\alpha, \beta, \gamma \in[0,1 / 3]$.

(c) $d_{F_{x y z} F_{u v w}} \leq \alpha d_{g x g u}+\beta d_{g y g v}+\gamma d_{g z g w}$ for some $\alpha, \beta, \gamma \in[0,1)$ such that $\alpha+\beta+\gamma<1$.

Then there exists a unique $x \in X$ such that $x=g x=F_{x x x}$. 
Proof (a) Consider $M^{c}$ defined as in Example 7. As $(X, d)$ is complete, then $\left(X, M^{c}, \min \right)$ is a complete FMS. Fix $x, y, z, u, v, w \in X$ and $t>0$, and we are going to prove (1) using $a=b=$ $c=1 / 3$ and $*=\min$. If $M_{g x g u}^{c}(t)=0$ or $M_{g y g v}^{c}(t)=0$ or $M_{g z g w}^{c}(t)=0$, then (1) is obvious. Suppose that $M_{g x g u}^{c}(t)=1, M_{g y g v}^{c}(t)=1$ and $M_{g z g w}^{c}(t)=1$. This means that $d_{g x g u}<t, d_{g y g v}<t$ and $d_{g z g w}<t$. Therefore, $t>\max \left(d_{g x g u}, d_{g y g v}, d_{g z g w}\right)$ and $k t>k \max \left(d_{g x g u}, d_{g y g v}, d_{g z g w}\right) \geq d_{F_{x y z} F_{u v w}}$. Hence, $M_{F_{x y z} F_{u v w}}^{c}(k t)=1$ and (1) is also true.

(b) In this case,

$$
\begin{aligned}
d_{F_{x y z} F_{u v w}} & \leq k\left(\alpha d_{g x g u}+\beta d_{g y g v}+\gamma d_{g z g w}\right) \leq k\left(\frac{1}{3} d_{g x g u}+\frac{1}{3} d_{g y g v}+\frac{1}{3} d_{g z g w}\right) \\
& =\frac{k}{3}\left(d_{g x g u}+d_{g y g v}+d_{g z g w}\right) \leq \frac{k}{3} 3 \max \left(d_{g x g u}, d_{g y g v}, d_{g z g w}\right) \\
& =k \max \left(d_{g x g u}, d_{g y g v}, d_{g z g w}\right) .
\end{aligned}
$$

(c) If $k=\alpha+\beta+\gamma<1$,

$$
\begin{aligned}
d_{F_{x y z} F_{u v w} \leq} & \alpha d_{g x g u}+\beta d_{g y g v}+\gamma d_{g z g w} \leq \alpha \max \left(d_{g x g u}, d_{g y g v}, d_{g z g w}\right) \\
& +\beta \max \left(d_{g x g u}, d_{g y g v}, d_{g z g w}\right)+\gamma \max \left(d_{g x g u}, d_{g y g v}, d_{g z g w}\right) \\
= & (\alpha+\beta+\gamma) \max \left(d_{g x g u}, d_{g y g v}, d_{g z g w}\right)=k \max \left(d_{g x g u}, d_{g y g v}, d_{g z g w}\right) .
\end{aligned}
$$

Example 16 If $X=\mathbb{R}, d(x, y)=|x-y|$ for all $x, y \in \mathbb{R}$ and $a, b, c, d, M \in \mathbb{R}$ are such that $M>$ $|a|+|b|+|c|$, the mappings $F: \mathbb{R}^{3} \rightarrow \mathbb{R}$ and $g: \mathbb{R} \rightarrow \mathbb{R}$, defined as $F_{x y z}=(a x+b y+c z+d) / M$ and $g x=x$ for all $x, y, z \in \mathbb{R}$, verify the hypothesis of Theorem 15 (c). It is easy to check that $\left(x_{0}, x_{0}, x_{0}\right)$, where $x_{0}=d /(M-a-b-c)$, is the unique tripled coincidence point of $F$ and $g$ and verifies $F\left(x_{0}, x_{0}, x_{0}\right)=x_{0}$.

Now, we prove the existence of a coupled coincided point for $F: X^{2} \rightarrow X$ and $g$ that generalizes Theorem 4.1 in [7], taking $a=b=1 / 2$. That is, the main result of the paper also covers the main theoretical results of Zhu and Xiao [7].

Corollary 17 Let $*$ be a $t$-norm of $H$-type such that $s * t \geq$ st for all $s, t \in[0,1]$. Let $k \in(0,1)$ and $a, b \in[0,1]$ be real numbers such that $a+b \leq 1$, let $(X, M, *)$ be a complete FMS and let $F: X^{2} \rightarrow X$ and $g: X \rightarrow X$ be two mappings such that $F\left(X^{2}\right) \subseteq g(X)$ and $g$ is continuous and commuting with $F$. Suppose that

$$
M_{F_{x y} F_{u v}}(k t) \geq M_{g x g u}(t)^{a} * M_{g y g v}(t)^{b}
$$

for all $x, y, u, v \in X$ and all $t>0$. Then there exists a unique $x \in X$ such that $x=g x=F_{x x}$.

Proof Define $c=0$ and $F^{\prime}: X^{3} \rightarrow X$ as $F_{x y z}^{\prime}=F_{x y}$ for all $x, y, z \in X$. Then $F^{\prime}\left(X^{3}\right)=F\left(X^{2}\right) \subseteq$ $g(X)$ and $F^{\prime}$ is commuting with $g\left(g F_{x y z}^{\prime}=g F_{x y}=F_{g x g y}=F_{g x g y g z}^{\prime}\right)$. Furthermore,

$$
\begin{aligned}
M_{F_{x y z}^{\prime} F_{u v w}^{\prime}}(k t) & =M_{F_{x y} F_{u v}}(k t) \geq M_{g x g u}(t)^{a} * M_{g y g v}(t)^{b} \\
& =M_{g x g u}(t)^{a} * M_{g y g \nu}(t)^{b} * 1 \geq M_{g x g u}(t)^{a} * M_{g y g v}(t)^{b} * M_{g z g w}(t)^{c} .
\end{aligned}
$$

Then there exists a unique $x \in X$ such that $g x=F_{x x x}^{\prime}$. If $y \in X$ verifies $F_{y y}=g y$, then $g y=$ $F_{y y}=F_{y y y}^{\prime}$, so $x=y$. 
Corollary 18 ([1, Theorem 2.2]) Let $(X, d)$ be a complete metric space and let $F: X^{2} \rightarrow X$ and $g: X \rightarrow X$ be two mappings such that $F\left(X^{2}\right) \subseteq g(X)$ and $g$ is continuous and commuting with $F$. Suppose that $F$ and $g$ verify some of the following conditions for all $x, y, u, v \in X$ :

(a) $d_{F_{x y} F_{u v}} \leq k \max \left(d_{g x g u}, d_{g y g v}\right)$ for some $k \in(0,1)$.

(b) $d_{F_{x y} F_{u v}} \leq k\left(\alpha d_{g x g u}+\beta d_{g y g v}\right)$ for some $k \in(0,1)$ and some $\alpha, \beta \in[0,1 / 2]$.

(c) $d_{F_{x y} F_{u v}} \leq \alpha d_{g x g u}+\beta d_{g y g v}$ for some $\alpha, \beta, \gamma \in[0,1)$ such that $\alpha+\beta<1$.

Then there exists a unique $x \in X$ such that $x=g x=F_{x x}$.

Proof Similar to the proof of Theorem 15.

Remark 19 In fact, the previous result is proved for $X$, a partially ordered set in [1].

Moreover, from a similar procedure, we can deduce the celebrated Banach contraction principle (Theorem 1).

\section{Applications}

\section{Lipschitzian systems}

Let $f_{1}, f_{2}, f_{3}: \mathbb{R} \rightarrow \mathbb{R}$ be Lipschitzian mappings and let $\beta_{1}, \beta_{2}, \beta_{3} \in \mathbb{R}$ be real numbers. Define $h: \mathbb{R} \rightarrow \mathbb{R}$ as $h(x)=\beta_{1} f_{1}(x)+\beta_{2} f_{2}(x)+\beta_{3} f_{3}(x)$ for all $x \in \mathbb{R}$. Then $h$ is another Lipschitzian mapping and $k_{h} \leq\left|\beta_{1}\right| k_{f_{1}}+\left|\beta_{2}\right| k_{f_{2}}+\left|\beta_{3}\right| k_{f_{3}}$. Obviously, if $K=\left|\beta_{1}\right| k_{f_{1}}+\left|\beta_{2}\right| k_{f_{2}}+\left|\beta_{3}\right| k_{f_{3}}<1$, then $h$ is a contraction, so there exists a unique $x_{0} \in \mathbb{R}$ such that $h_{x_{0}}=x_{0}$.

Next, define $F: \mathbb{R}^{3} \rightarrow \mathbb{R}$ as $F_{x y z}=\beta_{1} f_{1}(x)+\beta_{2} f_{2}(y)+\beta_{3} f_{3}(z)$ for all $x, y, z \in \mathbb{R}$. It is clear that $F_{x x x}=h_{x}$ for all $x \in \mathbb{R}$. Furthermore,

$$
d\left(F_{x_{1} x_{2} x_{3}}, F_{y_{1} y_{2} y_{3}}\right) \leq \sum_{i=1}^{3}\left|\beta_{i}\right| f_{i}\left(x_{i}\right)-f_{i}\left(y_{i}\right)\left|\leq \sum_{i=1}^{3}\right| \beta_{i}\left|k_{f_{i}}\right| x_{i}-y_{i} \mid \leq K \max _{1 \leq j \leq 3} d\left(x_{j}, y_{j}\right) .
$$

If $K<1$, then $F$ verifies (1) with $g x=x$ for all $x \in \mathbb{R}$.

Corollary 20 Let $f_{1}, f_{2}, f_{3}: \mathbb{R} \rightarrow \mathbb{R}$ be Lipschitzian mappings on $\mathbb{R}$ (provided with the Euclidean metric) and let $\beta_{1}, \beta_{2}, \beta_{3} \in \mathbb{R}$ such that $\left|\beta_{1}\right| k_{f_{1}}+\left|\beta_{2}\right| k_{f_{2}}+\left|\beta_{3}\right| k_{f_{3}}<1$. Then the system

$$
(S) \quad\left\{\begin{array}{l}
\beta_{1} f_{1}(x)+\beta_{2} f_{2}(y)+\beta_{3} f_{3}(z)=x \\
\beta_{1} f_{1}(y)+\beta_{2} f_{2}(z)+\beta_{3} f_{3}(x)=y \\
\beta_{1} f_{1}(z)+\beta_{2} f_{2}(x)+\beta_{3} f_{3}(y)=z
\end{array}\right.
$$

has a unique solution, which is $\left(x_{0}, x_{0}, x_{0}\right)$, where $x_{0}$ is the only real solution of $\beta_{1} f_{1}(x)+$ $\beta_{2} f_{2}(x)+\beta_{3} f_{3}(x)=x$.

Example 21 Consider the system

$$
\left\{\begin{array}{l}
30 \sin x-\frac{28}{1+y^{2}}+150=72 x-15 \arctan z \\
30 \sin y-\frac{28}{1+z^{2}}+150=72 y-15 \arctan x \\
30 \sin z-\frac{28}{1+x^{2}}+150=72 z-15 \arctan y
\end{array}\right.
$$


If we choose $f_{1}(x)=5+\sin x, f_{2}(x)=1 /\left(1+x^{2}\right)$ and $f_{3}(x)=\arctan x$, then $f_{1}, f_{2}$ and $f_{3}$ are Lipschitzian mappings, and $k_{f_{1}}=k_{f_{3}}=1$ and $k_{f_{2}}=3 \sqrt{3} / 8$. Let $\beta_{1}=5 / 12, \beta_{2}=-7 / 18$ and $\beta_{3}=5 / 24$. Then $\left|\beta_{1}\right| k_{f_{1}}+\left|\beta_{2}\right| k_{f_{2}}+\left|\beta_{3}\right| k_{f_{3}}=(30+7 \sqrt{3}) / 48<1$. As system $\left(S_{1}\right)$ is equal to $(S)$, then $\left(S_{1}\right)$ has a unique solution, which is of the form $\left(x_{0}, x_{0}, x_{0}\right)$, where $x_{0}$ is the only solution of

$$
30 \sin x-\frac{28}{1+x^{2}}+150=72 x-15 \arctan x .
$$

Finding, for example, the root by the bisection method, we get, approximately, $x_{0}=$ 2.5212648363927 .

\section{An integral system}

Let $a, b \in \mathbb{R}$ with $a<b$ and let $I=[a, b]$. Consider $X=\mathcal{L}^{1}(I)$ with the distance $d_{1}(f, g)=$ $\int_{I}|f(t)-g(t)| d t$, where $\int$ represents the Lebesgue integral. It is well known that $\left(\mathcal{L}^{1}(I), d_{1}\right)$ is a complete MS. Let $k, \beta_{1}, \beta_{2}, \beta_{3} \in \mathbb{R}$ be real numbers and let $G: \mathbb{R}^{3} \rightarrow \mathbb{R}$ be a mapping verifying $G(0,0,0)=0$ and

$$
\left|G_{x_{1} x_{2} x_{3}}-G_{y_{1} y_{2} y_{3}}\right| \leq k \sum_{i=1}^{3} \beta_{i}\left|x_{i}-y_{i}\right| \quad \text { for all }\left(x_{1}, x_{2}, x_{3}\right),\left(y_{1}, y_{2}, y_{3}\right) \in \mathbb{R}^{3} .
$$

If $A \in \mathbb{R}$, we want to find functions $f_{1}, f_{2}, f_{3} \in \mathcal{L}^{1}(I)$ such that

$$
f_{i}(x)=A+\int_{[a, x]} G\left(f_{i}(t), f_{i+1}(t), f_{i+2}(t)\right) d t
$$

holds for all $x \in I, i=1,2,3$.

For all $f_{1}, f_{2}, f_{3} \in \mathcal{L}^{1}(I)$ and all $x \in I$, define

$$
F_{f_{1} f_{2} f_{3}}(x)=A+\int_{[a, x]} G\left(f_{1}(t), f_{2}(t), f_{3}(t)\right) d t .
$$

On the one hand, it is not difficult to prove that $F_{f_{1} f_{2} f_{3}} \in \mathcal{L}^{1}(I)$, hence $F: \mathcal{L}^{1}(I)^{3} \rightarrow \mathcal{L}^{1}(I)$ is well defined. On the other hand,

$$
\begin{aligned}
d_{1}\left(F_{f_{1} f_{2} f_{3}}, F_{g_{1} g_{2} g_{3}}\right) & =\int_{I}\left|F_{f_{1} f_{2} f_{3}}(x)-F_{g_{1} g_{2} g_{3}}(x)\right| d x \\
& \leq \int_{I}\left(\int_{[a, x]}\left|G\left(f_{1}(t), f_{2}(t), f_{3}(t)\right)-G\left(g_{1}(t), g_{2}(t), g_{3}(t)\right)\right| d t\right) d x \\
& \leq \int_{I}\left(\int_{[a, x]} k \sum_{i=1}^{3} \beta_{i}\left|f_{i}(t)-g_{i}(t)\right| d t\right) d x \\
& \leq k \sum_{i=1}^{3} \beta_{i} \int_{I}\left(\int_{I}\left|f_{i}(t)-g_{i}(t)\right| d t\right) d x \\
& =k \sum_{i=1}^{3} \beta_{i} \int_{I} d_{1}\left(f_{i}, g_{i}\right) d x=k(b-a) \sum_{i=1}^{3} \beta_{i} d_{1}\left(f_{i}, g_{i}\right) .
\end{aligned}
$$

If we suppose that $K=k(b-a)\left(\beta_{1}+\beta_{2}+\beta_{3}\right)<1$, then $F$ verifies (1) with $g(f)=f$ for all $f \in \mathcal{L}^{1}(I)$. Then the system (23) has a unique solution, which is of the form $\left(f_{0}, f_{0}, f_{0}\right)$, where 
$f_{0} \in \mathcal{L}^{1}(I)$ is the only solution of the equation

$$
f_{0}(x)=A+\int_{[a, x]} G\left(f_{0}(t), f_{0}(t), f_{0}(t)\right) d t \quad \text { for all } x \in I
$$

(this exists as a simple application of the Banach contraction principle).

\section{Competing interests}

The authors declare that they have no competing interests.

\section{Authors' contributions}

All authors completed the paper together. All authors read and approved the final manuscript.

\section{Author details}

1 Department of Statistics and Operations Research, University of Jaén, Jaén, Spain. ${ }^{2}$ Department of Mathematics,

University of Jaén, Jaén, Spain. ${ }^{3}$ Department of Statistics and Operations Research, University of Granada, Granada, Spain.

Received: 1 July 2012 Accepted: 11 January 2013 Published: 11 February 2013

\section{References}

1. Bhaskar, T, Lakshmikantham, V: Fixed point theorems in partially ordered metric spaces and applications. Nonlinear Anal. TMA 65(7), 1379-1393 (2006)

2. Lakshmikantham, V, Ćirić, L: Coupled fixed point theorems for nonlinear contractions in partially ordered metric spaces. Nonlinear Anal. TMA 70, 4341-4349 (2009)

3. Fang, J: Common fixed point theorems of compatible and weakly compatible maps in Menger spaces. Nonlinear Anal. TMA 5-6, 1833-1843 (2009)

4. Shakeri, S, Ćirić, L, Saadati, R: Common fixed point theorem in partially ordered L-fuzzy metric spaces. Fixed Point Theory Appl. 2010, Article ID 125082 (2010)

5. Sedghi, S, Altun, I, Shobe, N: Coupled fixed point theorems for contractions in fuzzy metric spaces. Nonlinear Anal. TMA 72(3-4), 1298-1304 (2010)

6. Hu, X: Common coupled fixed point theorems for contractive mappings in fuzzy metric spaces. Fixed Point Theory Appl. 2011, Article ID 363716 (2011)

7. Zhu, X, Xiao, J: Note on "Coupled fixed point theorems for contractions in fuzzy metric spaces". Nonlinear Anal. TMA 74(16), 5475-5479 (2011)

8. Sintunavarat, W, Cho, Y, Kumam, P: Coupled coincidence point theorems for contractions without commutative condition in intuitionistic fuzzy normed spaces. Fixed Point Theory Appl. 2011, 81 (2011)

9. Zadeh, L: Fuzzy sets. Inf. Control 8, 338-353 (1965)

10. George, A, Veeramani, P: On some results in fuzzy metric spaces. Fuzzy Sets Syst. 64, 395-399 (1994)

11. Kramosil, I, Michalek, J: Fuzzy metric and statistical metric spaces. Kybernetika 11, 326-333 (1975)

12. Grabiec, M: Fixed points in fuzzy metric spaces. Fuzzy Sets Syst. 27(3), 385-389 (1988)

13. Fang, J: On fixed point theorems in fuzzy metric spaces. Fuzzy Sets Syst. 46, 107-113 (1992)

14. Cho, Y: Fixed points in fuzzy metric spaces. J. Fuzzy Math. 5(4), 949-962 (1997)

15. Gregori, V, Sapena, A: On fixed-point theorems in fuzzy metric spaces. Fuzzy Sets Syst. 125(2), 245-252 (2002)

16. Beg, I, Abbas, M: Common fixed points of Banach operator pair on fuzzy normed spaces. Fixed Point Theory 12(2), 285-292 (2011)

17. Elagan, SK, Rahmat, MS: Some fixed points theorems in locally convex topology generated by fuzzy $n$-normed spaces. Iran. J. Fuzzy Syst. 9(4), 43-54 (2012)

18. Berinde, $V$, Borcut, M: Tripled fixed point theorems for contractive type mappings in partially ordered metric spaces. Nonlinear Anal. TMA 74, 4889-4897 (2011)

19. Roldán, A, Martinez-Moreno, J, Roldán, C: Multidimensional fixed point theorems in partially ordered metric spaces. J. Math. Anal. Appl. 396, 536-545 (2012)

20. Hadžić, O, Pap, E: Fixed Point Theory in Probabilistic Metric Spaces. Kluwer Academic, Dordrecht (2001)

21. Borcut, $M$, Berinde, $\mathrm{V}$ : Tripled coincidence theorems for contractive type mappings in partially ordered metric spaces. Appl. Math. Comput. 218(10), 5929-5936 (2012)

doi:10.1186/1687-1812-2013-29

Cite this article as: Roldán et al.: Tripled fixed point theorem in fuzzy metric spaces and applications. Fixed Point Theory and Applications 2013 2013:29. 